\title{
Payments and freedoms: Effects of monetary and legal incentives on COVID-19 vaccination intentions in Germany
}

\author{
Philipp Sprengholz ${ }^{1 *}$, Luca Henkel ${ }^{2}$, and Cornelia Betsch ${ }^{1,3}$
}

\author{
${ }^{1}$ Media and Communication Science, University of Erfurt, Erfurt, Germany \\ ${ }^{2}$ University of Bonn, Bonn, Germany \\ ${ }^{3}$ Center for Empirical Research in Economics and Behavioral Sciences, University of Erfurt, \\ Erfurt, Germany
}

*Corresponding author: Philipp Sprengholz University of Erfurt

Nordhäuser Str. 63

99089 Erfurt

Germany

E-Mail: philipp.sprengholz@uni-erfurt.de

Phone: +49 1775986570

Funding. This work was supported by German Research Foundation (BE3970/12-1), Federal Centre for Health Education, Robert Koch Institute, Leibniz Institute for Psychology, Klaus Tschira Foundation, and University of Erfurt (no award/grant numbers).

Conflicts of Interest. None declared.

Acknowledgments. The study was conducted as part of Germany's COVID-19 Snapshot Monitoring (COSMO), a joint project of the University of Erfurt (Cornelia Betsch [PI], Lars Korn, Philipp Sprengholz, Philipp Schmid, Lisa Felgendreff, Sarah Eitze), the Robert Koch Institute (RKI; Lothar H. Wieler, Patrick Schmich), the Federal Centre for Health Education (BZgA; Heidrun Thaiss, Freia De Bock), the Leibniz Institute of Psychology (ZPID; Michael Bosnjak), the Science Media Center (SMC; Volker Stollorz), the Bernhard Nocht Institute for Tropical Medicine (BNITM; Michael Ramharter), and the Yale Institute for Global Health (Saad Omer).

Author contributions. PS, LH, and CB designed the research. PS and LH performed the research, planned and performed data analyses. PS wrote the initial draft, which was revised and approved by all authors.

Ethical Declaration. Our research obtained ethical clearance from the University of Erfurt's IRB (\#20200302/20200501), and all participants provided informed consent prior to data collection. 


\begin{abstract}
Monetary and legal incentives have been proposed to promote COVID-19 vaccination uptake. To evaluate the suitability of incentives, an experiment with German participants examined the effects of payments (varied within subjects: 0 to 10,000 EUR) and freedoms (varied between subjects: vaccination leading vs. not leading to the same benefits as a negative test result) on the vaccination intentions of previously unvaccinated individuals $(n=$ 782). While no effect could be found for freedoms, the share of participants willing to be vaccinated increased with the payment amount. However, a significant change required large rewards of 3,250 EUR or more. While monetary incentives could increase vaccination uptake by a few percentage points, the high costs of implementation challenge the efficiency of the measure and call for alternatives. As experimental data suggest that considering vaccination as safe, necessary, and prosocial increases an individual's likelihood of wanting to get vaccinated without payment, educational campaigns should emphasize these features when promoting vaccination against COVID-19.
\end{abstract}




\section{Introduction}

Rapid, large-scale uptake of vaccines against COVID-19 is required to control and eventually end the current pandemic. However, vaccine hesitancy may prevent a significant share of the population from getting vaccinated (Attwell et al., 2021). Previous research indicates that low vaccination intentions can result from people having little confidence in vaccine safety, being complacent (i.e., considering vaccination as rather unnecessary) or calculative (i.e., extensively weighing risks and benefits), encountering barriers constraining vaccination, or perceiving low collective responsibility (e.g., lack of willingness to get vaccinated to protect others) (Betsch et al., 2018). Researchers have discussed various interventions for addressing these antecedents of vaccination and increasing vaccination intentions, ranging from information campaigns to mandatory vaccination (Betsch, Böhm, Korn, \& Holtmann, 2017; Sprengholz, Felgendreff, Böhm, \& Betsch, 2021). As incentives have been shown to promote certain health behaviors, such as maintaining a healthier diet and quitting smoking (Gardiner \& Bryan, 2017; Notley et al., 2019), offering rewards for vaccination could bolster vaccination intentions as well. This may be done in different ways. First, monetary incentives could be used, reimbursing people for the time needed to get vaccinated and to recover from possible side effects. For instance, some companies in the United States and Germany have started offering employees one-time payments when they get vaccinated (Maruf, 2021). While a hypothetical experiment with German participants at the end of 2020 suggested that payments of up to 200 EUR (about 240 USD) for getting vaccinated did not increase people's intentions to get vaccinated (Sprengholz, Eitze, Felgendreff, Korn, \& Betsch, 2021), a different picture emerged in a US study, where vaccination intentions increased by 13.6 percentage points after individuals were offered a payment of 500 USD (Serra Garcia \& Szech, 2021). While the effect of monetary incentives certainly depends on the local health system and cultural background, the results indicate that 
monetary incentives exceeding mere reimbursement of vaccination-related costs may bolster vaccination intentions. Second, legal incentives could be employed. As vaccinated individuals are unlikely to transmit the disease (Levine-Tiefenbrun et al., 2021), they could enjoy more rights and freedoms and be less constrained by COVID-19 regulations compared to unvaccinated people. For instance, allowing vaccinated individuals to enter shops, get haircuts, or attend certain events without having to get tested may drive vaccination intentions (Wilf-Miron, Myers, \& Saban, 2021).

While researchers have discussed the ethics of both monetary and legal incentives for COVID-19 vaccine uptake (Brown, Savulescu, Williams, \& Wilkinson, 2020; Jecker, 2021; Largent \& Miller, 2021; Savulescu, 2020), we conducted a survey experiment investigating their single and combined potential effects on vaccination intentions. The evidence can help in weighing the benefits and costs of monetary and legal incentives and, thus, can inform the efficient design of future vaccination policies.

\section{Methods}

\section{Participants and design}

The experiment was conducted on April 20-21, 2021, as part of the COVID-19 Snapshot Monitoring (COSMO) cross-sectional study series (Betsch, Wieler, \& Habersaat, 2020). Participants were recruited from a non-probabilistic German sample $(N=997)$, which was quota-representative for age $\times$ gender and federal state. Excluding participants who had already been vaccinated against COVID-19 $(n=215)$ yielded a final sample of $n=782$. Participants ranged in age from 18 to 74 years $(M=44.01, S D=15.66)$ and included 376 males and 406 females. They were randomly assigned to one of two experimental conditions (legal incentive vs. no legal incentive), and financial incentives were manipulated within subjects.

\section{Procedure and materials}


We assessed relevant demographic information, financial worries, and psychological antecedents of vaccination for explorative purposes. After participants had read a scenario based on the legal incentive condition, they repeatedly decided between not getting vaccinated and getting vaccinated with an incentive ranging from 0 to 10,000 EUR.

\section{Financial worries}

Participants were asked how worried they are about getting into financial trouble over losing money due to the pandemic. Answers were assessed on a scale ranging from 1 (not worried at all) to 7 (very much worried).

\section{Psychological antecedents of vaccination}

Participants were asked to think about a COVID-19 vaccine that was officially recommended for them. An adapted version of the 5C short scale (Betsch et al., 2018) was used to assess confidence (I am completely confident that the COVID-19 vaccine is safe), complacency (Vaccination against COVID-19 is unnecessary because COVID-19 is not common anymore), constraints (Everyday stress prevents me from getting vaccinated against COVID-19), calculation (When I think about getting vaccinated against COVID-19, I weigh benefits and risks to make the best decision possible), and collective responsibility (When everyone is vaccinated against COVID-19, I don't have to get vaccinated too) in relation to the vaccine. Items were rated on a scale ranging from 1 (strongly disagree) to 7 (strongly agree). Scores for collective responsibility were reversed before analyses.

\section{Experimental manipulation}

At the time of the experiment, legal regulations in Germany required everyone to wear face masks in public areas, such as city centers, and it was mandatory to have a negative coronavirus test to attend cultural events (when permitted at all) or to access services, such as haircuts. Participants in the legal incentive condition were asked to imagine that being vaccinated would lead to more rights in those areas, such as being allowed to discontinue 
wearing face masks and not needing a test to attend cultural events or access services. In the no legal incentive condition, participants were told that getting vaccinated would not result in additional freedoms.

\section{Vaccination decisions}

A price list design was used to determine participants' vaccination intentions and payment preferences. Price lists are a standard method for measuring the effects of monetary incentives, since they are easy to explain and implement (Andersen, Harrison, Lau, \& Rutström, 2006). In a series of repeated decisions, participants chose between two options: not getting vaccinated vs. getting vaccinated and being paid a specific amount. Amounts from 0 EUR to 5,000 EUR (in increments of 250 EUR) and 10,000 EUR were offered, resulting in 22 binary decisions for each participant.

\section{Results}

\section{Effects of legal and monetary incentives on vaccination intentions}

Figure 1 displays the fraction of participants willing to get vaccinated for each monetary amount offered in the price list. Legal incentives had virtually no impact on vaccination intentions. Without payment (0 EUR), $61.4 \%$ of participants in the no legal incentive condition and $65.1 \%$ in the legal incentive condition were willing to get vaccinated; the difference was not significant ( $p=.300$; two-sided Fisher's exact test). Similarly, differences were not significant for every other monetary amount except for 10,000 EUR. Interestingly, the share of people willing to get vaccinated for this large reward was higher when no legal incentives were offered ( $p=0.043$; two-sided Fisher's exact test). Notably, however, the latter significant difference was not robust to the exclusion of 84 participants with non-monotone vaccination intentions - that is, those who opted for vaccination at some amount but switched to non-vaccination at a higher amount (see online supplement). 


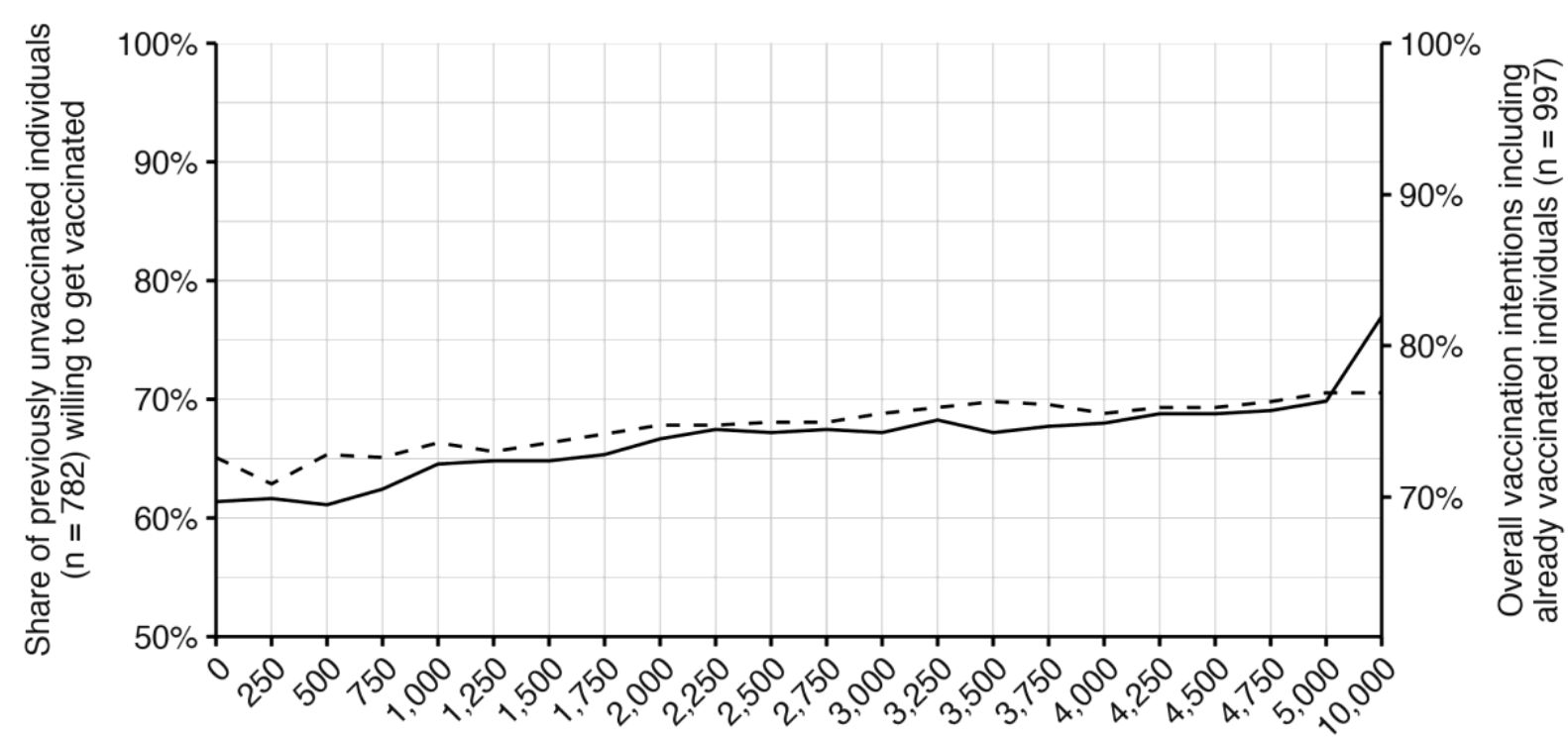

Compensation [EUR]

\section{Figure 1. Willingness to get vaccinated by legal and monetary incentives.}

Note: While legal incentives had virtually no impact on vaccination intentions, monetary incentives of 3,250 EUR and above led to a significant increase of people willing to be vaccinated against COVID-19 (compared to 0 EUR). As a reference, the fraction on the right also includes the $n=215$ participants that had already been vaccinated and were not included in the experiment.

Monetary incentives increased participants' willingness to get vaccinated, but for significant increases, large amounts were needed. At the 5\% level, a significant difference compared to the 0 EUR benchmark was only reached at 3,250 EUR and above. When presented with the maximum offer of 10,000 EUR, the share of people willing to get vaccinated increased by 10.4 percentage points compared to when no money was offered.

\section{Determinants of getting vaccinated with and without monetary incentives}

To investigate factors associated with the decision to get vaccinated with or without monetary incentives, we divided the experimental sample into three groups: (1) participants not willing to get vaccinated regardless of whether payment was offered $(n=144),(2)$ participants willing to get vaccinated without payment $(n=495)$, and (3) participants willing to get vaccinated only when payment was offered $(n=143)$. A multinomial logistic regression was performed to investigate differences among the three groups regarding age, 
gender, financial worries, the $5 \mathrm{C}$, and the impact of legal incentives (Table 1), further controlling for household size and income, education, and migration background (for complete results, see Table $\mathrm{S} 1$ in the online supplement).

Table 1: Determinants of getting vaccinated with and without monetary incentive

\begin{tabular}{|c|c|c|c|c|}
\hline \multirow{2}{*}{ Predictors } & \multicolumn{2}{|c|}{$\begin{array}{l}\text { Getting vaccinated without } \\
\text { monetary incentive }\end{array}$} & \multicolumn{2}{|c|}{$\begin{array}{c}\text { Getting vaccinated for monetary } \\
\text { incentive only }\end{array}$} \\
\hline & $O R$ & $95 \% C I$ & $O R$ & $95 \% C I$ \\
\hline (Constant) & 0.06 & $0.006-0.648$ & 1.13 & $0.146-8.715$ \\
\hline $\begin{array}{l}\text { Experimental manipulation: legal } \\
\text { incentive (Baseline: no legal } \\
\text { incentive) }\end{array}$ & 1.01 & $0.577-1.755$ & 0.62 & $0.362-1.059$ \\
\hline Age & 1.01 & $0.984-1.027$ & 0.98 & $0.959-0.999$ \\
\hline Gender: female (Baseline: male) & 0.63 & $0.347-1.129$ & 0.79 & $0.442-1.402$ \\
\hline Financial worries & 1.02 & $0.896-1.156$ & 1.02 & $0.913-1.156$ \\
\hline Confidence & 2.32 & $1.942-2.771$ & 1.49 & $1.257-1.769$ \\
\hline Complacency & 0.55 & $0.451-0.683$ & 0.81 & $0.688-0.957$ \\
\hline Calculation & 0.80 & $0.677-0.935$ & 0.99 & $0.851-1.159$ \\
\hline Constraints & 1.09 & $0.879-1.358$ & 1.07 & $0.894-1.289$ \\
\hline Collective responsibility & 1.51 & $1.259-1.804$ & 0.99 & $0.851-1.151$ \\
\hline
\end{tabular}

Note: Results of the multinomial logistic regression analysis (Cox \& Snell's $R^{2}=.51$, Nagelkerke's $R^{2}=.61$ ). Both groups were compared to participants not willing to get vaccinated regardless of payment. Results were further controlled for household size and income, education, and migration background (for complete results, see supplemental Table S1). Bold values denote significant predictors with $p<.05$.

Participants who preferred being vaccinated (regardless of payment preferences) indicated higher levels of confidence and lower levels of complacency compared to those who did not want to get vaccinated. When comparing the two groups willing to get vaccinated, those who preferred payment showed less confidence and more complacency. Furthermore, those who were willing to get vaccinated without payment indicated less calculation and higher levels of collective responsibility compared to the other two groups. While there were no differences between the groups regarding gender and financial worries, 
younger participants preferred a financial incentive for vaccination. As before, legal incentives did not predict participants' vaccination decisions.

\section{Determinants of minimum required monetary incentives}

We further examined factors influencing the minimum required monetary incentive for participants who were willing to get vaccinated only if payment was offered $(n=143)$. Two linear regressions were conducted to regress the minimum accepted payment on age, gender, financial worries, the 5C, and legal incentives, again controlling for household size and income, education, and migration background. The two regressions dealt differently with participants who switched between non-vaccination and (paid) vaccination more than once ( $n$ $=28$ ). In the first regression (model 1), these participants were excluded; in the second regression (model 2), their first switching point was interpreted as their minimum accepted payment and served as a dependent variable. In both regressions, no significant effects could be found for gender, financial worries, and the 5C (see supplemental Table S2). However, in both regressions, larger payments were related to higher age (model 1: $\beta=0.24, b=60.08$, $S E=26.81,95 \% C I=[7.531 ; 112.619] ;$ model $2: \beta=0.26, b=60.76, S E=22.39,95 \% C I=$ $[16.885 ; 104.637])$ and not being offered legal incentives (model $1: \beta=-0.35, b=-2,620.53$, $S E=744.76,95 \% C I=[-4,080.235 ;-1,160.826] ;$ model $2: \beta=-0.37, b=-2,550.45, S E=$ $561.49,95 \% C I=[-3,650.939 ;-1,449.957])$. Furthermore, in model 2, stronger perceived constraints to getting vaccinated were related to higher monetary incentives $(\beta=0.20, b=$ $395.50, S E=198.72,95 \% C I=[6.007 ; 784.992])$.

\section{Extrapolated costs of monetary incentives}

As monetary incentives had a positive effect on vaccination intentions, we calculated implementation costs for the adult German population (18 years and older; about 70 million people) for different vaccination uptake rates (Figure 2). It would be possible to vaccinate about $70 \%$ of the adult population without payments, but increasing vaccination uptake to 
$80 \%$, as demanded by the World Health Organization (WHO, 2021), would require incentives worth at least 500 billion EUR.

- No legal incentive $\bullet$ Legal incentive

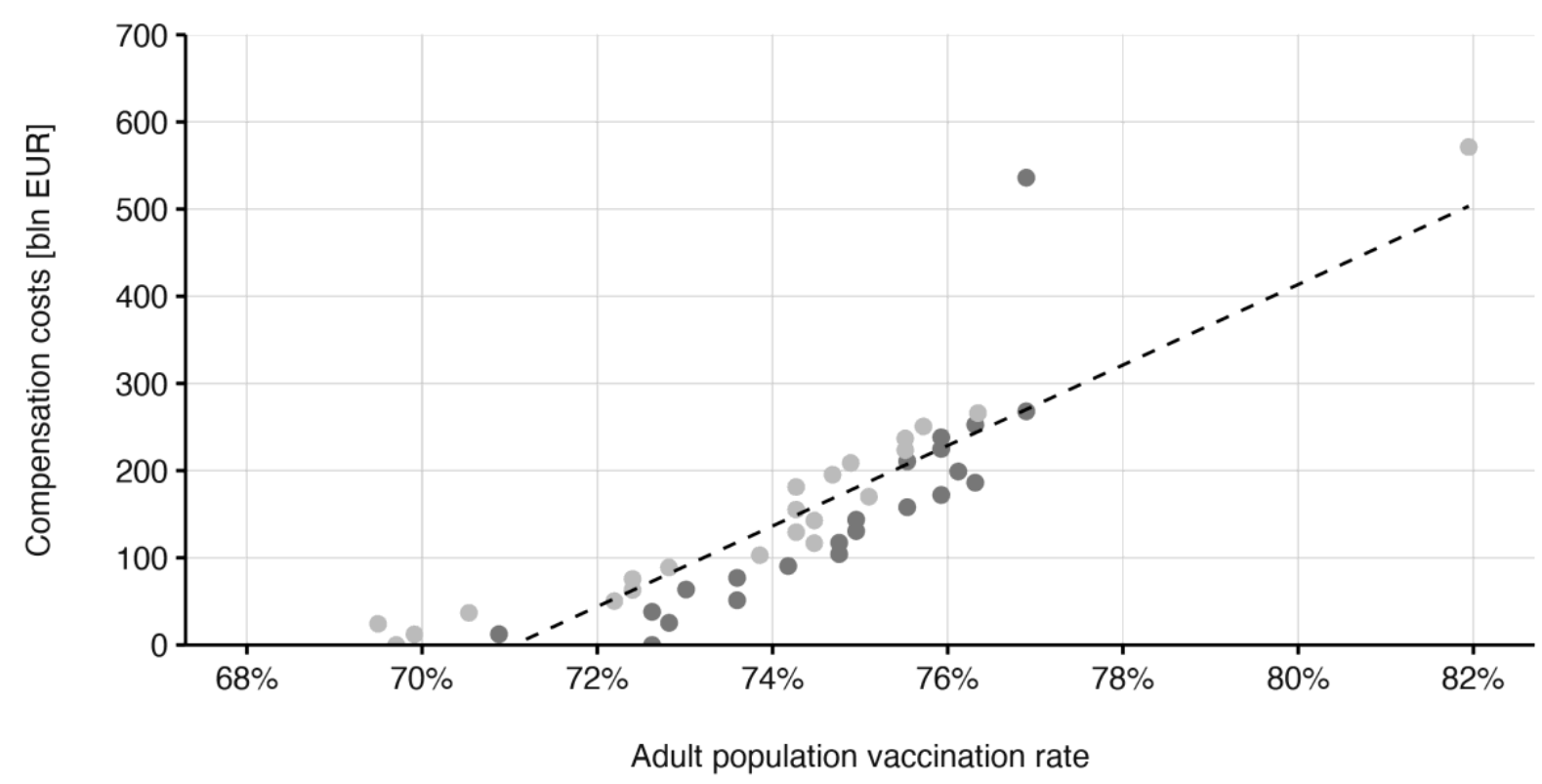

Figure 2. Economic costs associated with realizing specific vaccination uptake rates for the adult population in Germany.

Note: The visualization assumes that individuals 75 years and older will make decisions similar to the younger adults examined in our study. The dotted line denotes a linear fit after collapsing the legal incentive conditions.

\section{Discussion}

We investigated the effects of legal and monetary incentives on vaccination intentions. Our results indicate that legal incentives do not increase the willingness to vaccinate. However, this may be due to the specific incentives offered in the experiment, where vaccination was predominantly framed as a replacement for testing. Offering stronger incentives, such as being allowed to eat out, travel for leisure, or attend a music festival, could indeed boost vaccination intentions. While the majority of previously unvaccinated participants were willing to get vaccinated without a financial reward, about a fifth opted for vaccination only when a payment of up to 10,000 EUR was offered. Interestingly, compared 
to participants who were willing to get vaccinated regardless of payment, monetary incentives motivated less confident and more complacent participants to want the vaccine. Thus, people who think that vaccination is rather unnecessary and who are not entirely sure about the safety of vaccines could be motivated to get vaccinated when (high) monetary incentives are offered.

The WHO (2021) has urged countries to vaccinate at least $80 \%$ of their adult population as soon as feasible. Our results indicate that monetary incentives could help to achieve this rate. However, more people may need to be vaccinated when more contagious mutations of the virus emerge. In addition, immunizing children could become important for the same reasons. As previous research indicates that parents are more risk averse when contemplating their children's vaccination than their own, the impact of monetary incentives on these decisions may be small, calling for other measures to improve vaccine uptake.

Overall, the data revealed that high amounts need to be paid to make a difference in Germany. While Serra-Garcia and Szech (2021) showed that vaccination uptake among Americans could be leveraged by more than $10 \%$ by offering a payment of 500 USD, no such effect was apparent in the German sample, where an increase of 5\% was found to require 3,250 EUR. Therefore, the observed effects of legal and monetary incentives are likely to be different for other populations and cultural backgrounds and should be generalized with care. Moreover, the so-called compromise effect could be a methodological issue complicating a straightforward interpretation of our results (Andersen et al., 2006; Birnbaum, 1992; Simonson, 1989). It has been shown that when presented a price list, participants are perceptually drawn to the center of the price list, making those options appear more attractive. This could bias the elicited amounts. However, since an incentive of 200 EUR in another hypothetical experiment not using a price list design proved to be ineffective at increasing vaccination intentions in the German population (Sprengholz, Eitze, et al., 2021), 
it seems unlikely that this effect can explain our findings. Nevertheless, fictitious incentives and assessment of vaccination intentions may not offer a perfect representation of real-life vaccine decision-making. Although intention usually predicts behavior (Sheeran, 2002), there may be a gap between the two, especially as the social desirability of being rewarded for vaccination may be low. Therefore, the observed effects of legal and monetary incentives on vaccination intentions could be considered conservative estimates.

When large monetary incentives are needed to increase vaccination uptake, ethical and economic concerns arise. Incentives rob the act of vaccination of its moral significance (Largent \& Miller, 2021), possibly generating expectations of receiving payment for other vaccinations as well. Furthermore, large payments could increase vaccine hesitancy, because they may be perceived as compensation for severe adverse effects. Scholars also fear that large payments could be especially coercive to economically disadvantaged groups (Jecker, 2021). However, we could not find a link between financial worries and willingness to get vaccinated for a monetary reward. From an economic perspective, monetary incentives are only feasible if the benefits of higher vaccination rates outweigh the payment costs. In the case of Germany, increasing vaccination uptake by a few percentage points would require several hundred billion euros, challenging the efficiency of the measure.

Fortunately, our results suggest an alternative pathway to increasing vaccination uptake. As high levels of confidence and collective responsibility and low levels of complacency and calculation were related to willingness to be vaccinated without payment, improving these aspects should increase vaccination uptake at much lower costs. In any case, educational campaigns should highlight the safety and efficacy of vaccines. As previous research has shown, providing information about the prosocial impact of vaccination is important too (Betsch et al., 2017). When people realize that their own shots also protect those who cannot be vaccinated, such as children and individuals with an immunodeficiency, 
vaccination intentions increase. Recent research on influenza vaccination further indicates that scheduling appointments for shots and sending messages reminding individuals about vaccination opportunities prior to primary care visits could boost vaccination rates at low costs (Milkman et al., 2021). In conclusion, incentives may work, but the cost-benefit ratio seems questionable. Only if educational campaigns and nudges are insufficient to increase vaccination uptake, payments could add relevant percentage points, given thorough ethical embedment of the measure and sufficient monetary power.

\section{Online supplement}

Supplemental tables, data, and the data analysis script are available at https://osf.io/4kw2u/

\section{References}

Andersen, S., Harrison, G. W., Lau, M. I., \& Rutström, E. E. (2006). Elicitation using multiple price list formats. Experimental Economics, 9(4), 383-405. https://doi.org/10.1007/s10683-006-7055-6

Attwell, K., Lake, J., Sneddon, J., Gerrans, P., Blyth, C., \& Lee, J. (2021). Converting the maybes: Crucial for a successful COVID-19 vaccination strategy. PLOS ONE, 16(1), e0245907. https://doi.org/10.1371/journal.pone.0245907

Betsch, C., Böhm, R., Korn, L., \& Holtmann, C. (2017). On the benefits of explaining herd immunity in vaccine advocacy. Nature Human Behaviour, 1(3), 0056. https://doi.org/10.1038/s41562-017-0056

Betsch, C., Schmid, P., Heinemeier, D., Korn, L., Holtmann, C., \& Böhm, R. (2018). Beyond confidence: Development of a measure assessing the 5C psychological antecedents of vaccination. PLOS ONE, 13(12), e0208601. https://doi.org/10.1371/journal.pone.0208601

Betsch, C., Wieler, L. H., \& Habersaat, K. (2020). Monitoring behavioural insights related to COVID-19. The Lancet, 395(10232), 1255-1256. https://doi.org/10.1016/S0140- 
$6736(20) 30729-7$

Birnbaum, M. H. (1992). Violations of Monotonicity and Contextual Effects in Choice-Based Certainty Equivalents. Psychological Science, 3(5), 310-315. https://doi.org/10.1111/j.1467-9280.1992.tb00679.x

Brown, R. C. H., Savulescu, J., Williams, B., \& Wilkinson, D. (2020). Passport to freedom? Immunity passports for COVID-19. Journal of Medical Ethics, 46(10), 652-659. https://doi.org/10.1136/medethics-2020-106365

Gardiner, C. K., \& Bryan, A. D. (2017). Monetary Incentive Interventions Can Enhance Psychological Factors Related to Fruit and Vegetable Consumption. Annals of Behavioral Medicine, 51(4), 599-609. https://doi.org/10.1007/s12160-017-9882-4 Jecker, N. S. (2021). What money can't buy: an argument against paying people to get vaccinated. Journal of Medical Ethics, medethics-2021-107235. https://doi.org/10.1136/medethics-2021-107235

Largent, E. A., \& Miller, F. G. (2021). Problems With Paying People to Be Vaccinated Against COVID-19. JAMA, 325(6), 534. https://doi.org/10.1001/jama.2020.27121

Levine-Tiefenbrun, M., Yelin, I., Katz, R., Herzel, E., Golan, Z., Schreiber, L., ... Kishony, R. (2021). Initial report of decreased SARS-CoV-2 viral load after inoculation with the BNT162b2 vaccine. Nature Medicine. https://doi.org/10.1038/s41591-021-01316-7

Maruf, R. (2021). These companies are paying their employees to receive the Covid-19 vaccine. Retrieved May 3, 2021, from https://edition.cnn.com/2021/03/24/business/covid-vaccine-incentivescompanies/index.html

Milkman, K. L., Patel, M. S., Gandhi, L., Graci, H. N., Gromet, D. M., Ho, H., ... Duckworth, A. L. (2021). A megastudy of text-based nudges encouraging patients to get vaccinated at an upcoming doctor's appointment. Proceedings of the National Academy 
of Sciences, 118(20), e2101165118. https://doi.org/10.1073/pnas.2101165118

Notley, C., Gentry, S., Livingstone-Banks, J., Bauld, L., Perera, R., \& Hartmann-Boyce, J. (2019). Incentives for smoking cessation. Cochrane Database of Systematic Reviews. https://doi.org/10.1002/14651858.CD004307.pub6

Savulescu, J. (2020). Good reasons to vaccinate: mandatory or payment for risk? Journal of Medical Ethics, medethics-2020-106821. https://doi.org/10.1136/medethics-2020106821

Serra Garcia, M., \& Szech, N. (2021). Choice Architecture and Incentives Increase COVID19 Vaccine Intentions and Test Demand (No. 9003).

Sheeran, P. (2002). Intention-Behavior Relations: A Conceptual and Empirical Review. European Review of Social Psychology, 12(1), 1-36. https://doi.org/10.1080/14792772143000003

Simonson, I. (1989). Choice Based on Reasons: The Case of Attraction and Compromise Effects. Journal of Consumer Research, 16(2), 158. https://doi.org/10.1086/209205 Sprengholz, P., Eitze, S., Felgendreff, L., Korn, L., \& Betsch, C. (2021). Money is not everything: experimental evidence that payments do not increase willingness to be vaccinated against COVID-19. Journal of Medical Ethics, medethics-2020-107122. https://doi.org/10.1136/medethics-2020-107122

Sprengholz, P., Felgendreff, L., Böhm, R., \& Betsch, C. (2021). Vaccination Policy Reactance: Predictors, Consequences, and Countermeasures. Preprint. https://doi.org/10.31234/osf.io/98e4t

WHO Regional Office for Europe. (2021). Ad-hoc meeting of the European Technical Advisory Group of Experts on Immunization (ETAGE): virtual meeting, hosted in Copenhagen, Denmark, 28 April 2021.

Wilf-Miron, R., Myers, V., \& Saban, M. (2021). Incentivizing Vaccination Uptake. JAMA, 
325(15), 1503. https://doi.org/10.1001/jama.2021.4300 